
\title{
Clinical Cardiology
}

CASE REPORT

\section{A Rare Coronary Anomaly: Congenital Absence of the Left Circumflex Artery}

\section{Robert Tonks, MD*, Rajiv Sharma, MD and Raj Baljepally, MD, FACC}

Department of Cardiology, Heart Lung Vascular Institute, University of Tennessee Medical Center, USA

*Corresponding author: Robert Tonks, MD, Department of Cardiology, Heart Lung Vascular Institute, University of Tennessee Medical Center, 1924 Alcoa Hwy, Knoxville, TN 37920, USA, Tel: 1-865-111-2183

\begin{abstract}
Congenital anomalies of the coronary vasculature are usually asymptomatic and rare with an incidence of less than $1 \%$ and there are few cases reported of congenital absence of the left circumflex artery. It is important to recognize this anomaly and define the anatomy by left heart catheterization or coronary angiography by computerized tomography when considering percutaneous intervention or coronary artery bypass surgery on patients without a left circumflex artery.
\end{abstract}

\section{Keywords}

Anomalous coronary artery, Congenital anomaly, Left circumflex artery absence

\section{Manuscript}

71 yo male that recently moved to the area from out of state who presented to clinic complaining of increased fatigue, exertional chest pain and dyspnea that resolved with rest. His past medical history is significant for coronary artery disease with a drug eluting stent in the right coronary artery, hypertension, hyperlipidemia, diabetes and history of tobacco and alcohol use.

He had an abnormal stress test in the recent past but refused to have a cardiac cath at that time since his symptoms had temporarily improved. However, when

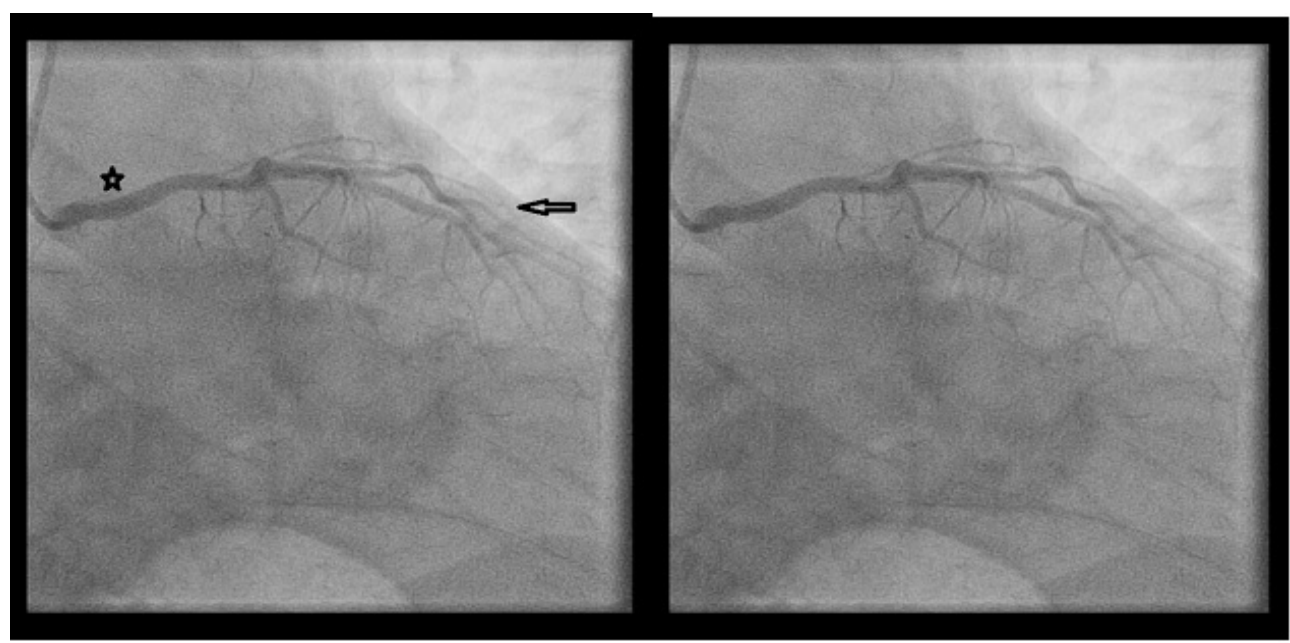

Figure 1: (Labeled and unlabeled).

The left main coronary artery (star) is elongated and gives rise to the left anterior descending artery and a substantial diagonal artery (arrow), but there is no left circumflex artery visible in this right anterior oblique, caudal view via cinematography during the left heart catheterization. Left Circumflex Artery. Int J Clin Cardiol 6:156. doi.org/10.23937/2378-2951/1410156

Accepted: August 24, 2019; Published: August 26, 2019

Copyright: (c) 2019 Tonks R, et al. This is an open-access article distributed under the terms of the Creative Commons Attribution License, which permits unrestricted use, distribution, and reproduction in any medium, provided the original author and source are credited. 


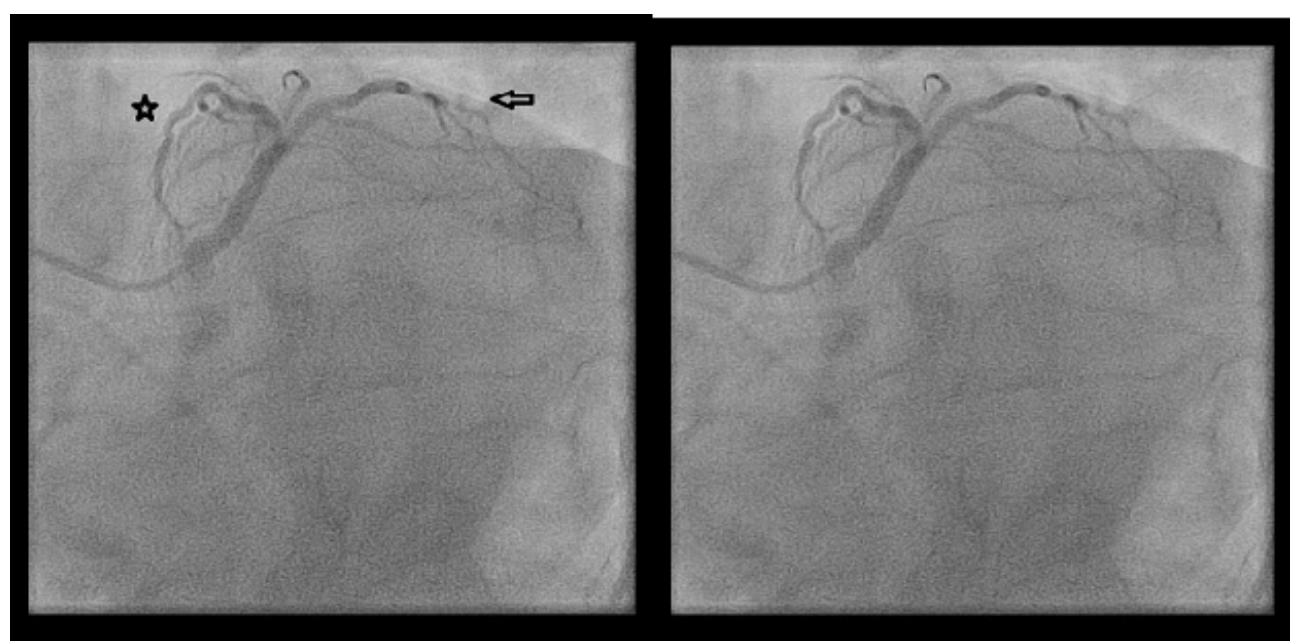

Figure 2: (Labeled and unlabeled).

An elongated left main coronary artery gives rise to the left anterior descending artery (star) which then gives off a substantial bifurcating diagonal branch (arrow) in the left anterior oblique, caudal ("spider") view via cinematography during the left heart catheterization.
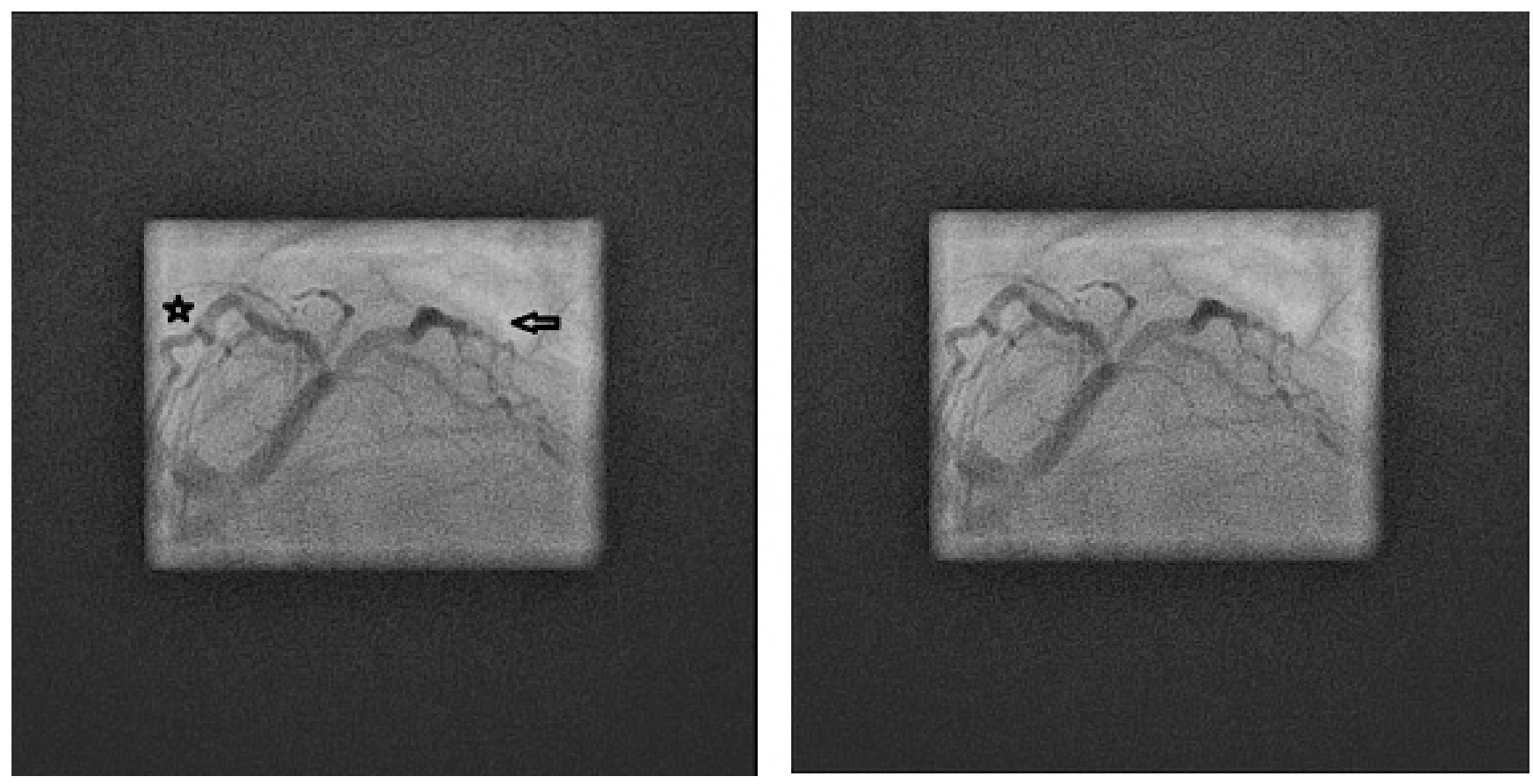

Figure 3: (Labeled and unlabeled).

This is an 8x magnification of the left anterior oblique, caudal ("spider") view via cinematography during the left heart catheterization, showing the left anterior descending artery (star) and large diagonal branch (arrow) but there is also no sign of a left circumflex stump or cleft that may suggest an occluded left circumflex branch.

he began having similar symptoms with exertion again, he returned to the clinic and was scheduled for diagnostic left heart catheterization. During the left heart catheterization, the patient was found to have a long left main coronary artery giving rise to with high grade stenosis in the ostium of the left anterior descending artery (Figure 1, Figure 2 and Figure 3 ) as well as a high grade stenosis in the right posterior lateral branch of the expansive right coronary artery network. The right coronary artery was very extensive, providing perfusion to a significant portion of the lateral myocardial wall (Figure 4). Multiple attempts were made with various catheters, multiple operators and several aortograms (Figure 5) were performed with no sign of the left circumflex artery. It was determined that the patient had a congenitally absent left circumflex artery with a super-dominate right coronary network to supplement the left coronary arteries.

\section{Comments}

Congenital anomalies of the coronary vasculature are usually asymptomatic and rare with an incidence of less than 1\% [1]. Complete absence of the left circumflex is an extremely rare finding with reported incidence 


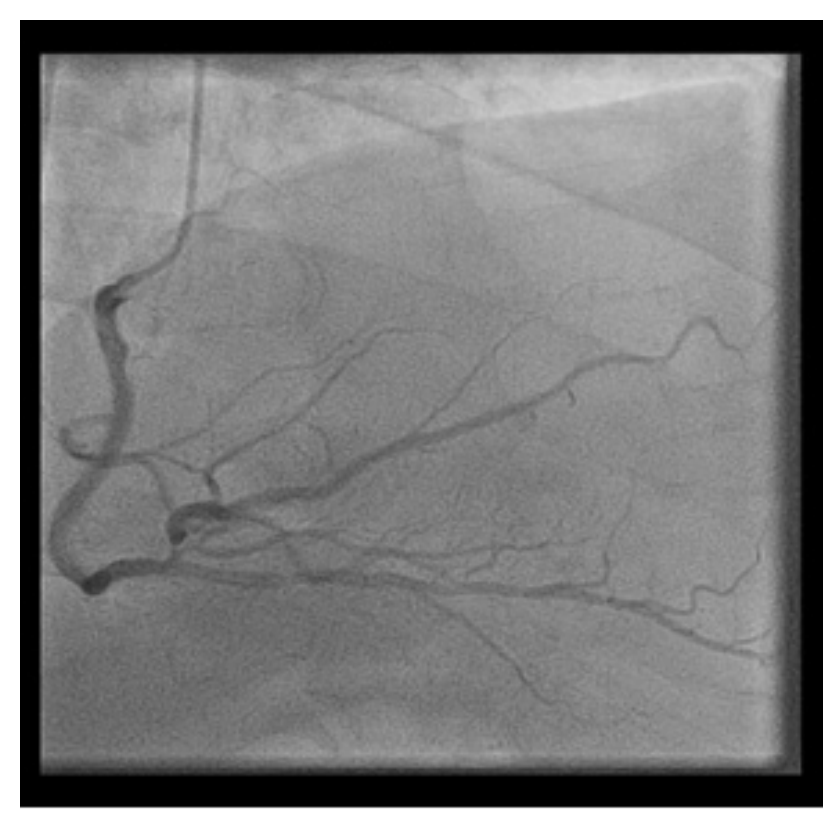

Figure 4: The right coronary artery is super dominate and expands to supply blood flow to the lateral wall that would be supplied by the absent left circumflex artery in this right anterior oblique, cranial view via cinematography during the left heart catheterization.

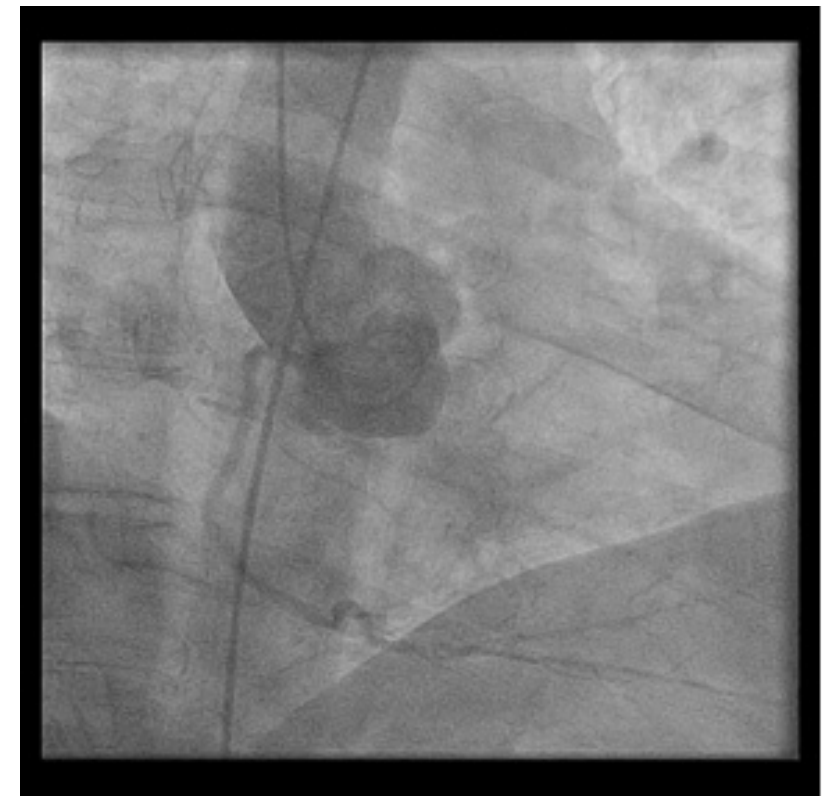

Figure 5: This image is of an aortogram performed via cinematography during left heart catheterization. There is some non-selective filling of the right coronary artery observed during the aortogram in the straight right anterior oblique view, but no sign of an anomalous left circumflex artery. of only $0.003 \%$ [2]. There are few cases reported of congenital absence of the left circumflex artery but in each case there is an elongated left main coronary artery (Figure 1), substantial diagonal branches from the left anterior descending artery (Figure 2 and Figure 3), and a super-dominate right coronary artery (Figure 4) to perfuse the lateral wall. It is important to recognize this anomaly and define the anatomy by left heart catheterization or coronary angiography by computerized tomography when considering percutaneous intervention or coronary artery bypass surgery on patients without a left circumflex artery [3].

\section{Disclaimer}

None.

\section{Conflicts of Interest}

None.

\section{References}

1. Moscucci M (2014) Grossman and Baim's cardiac catheterization, angiography, and intervention. ( $8^{\text {th }}$ edn), Lippincott Williams \& Wilkins, Philadelphia, 335-353.

2. Ali M, Hanley A, McFadden EP, Vaughan CJ (2011) Coronary artery anomalies: A practical approach to diagnosis and management. Heart Asia 3: 8-12.

3. Page HL, Engel HJ, Campbell WB, Thomas CS (1974) Anomalous origin of the left circumflex coronary artery: Recognition, angiographic demonstration and clinical significance. Circulation 50: 768-773.

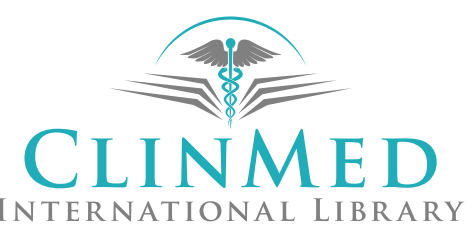

